\title{
DEVELOPMENT OF AN INTERACTIVE SOFTWARE TO STUDY ENERGETIC METABOLISM
}

\author{
Emanuella da Silva Cardoso ${ }^{1}$, Patrícia Burth ${ }^{1}$, Cátia Lacerda Sodré1 \\ ${ }^{1}$ Universidade Federal Fluminense, Biologia Celular e Molecular (RJ, Brasil)
}

INTRODUCTION: Technology allows the creation of dynamic interfaces and graphics, which enables the construction of different scenarios that simulate biochemical events at cellular and molecular level. Furthermore, games have the ability to amuse and stimulate students and thus keeping them interested and receptive. Therefore, digital games must be explored as teaching aids once they have features that enhance the teaching process. OBJECTIVES: This project aimed to develop an educational software that contributes to the understanding of various events taking place in energetic metabolism. MATERIALS AND METHODS: We developed a downloadable educational game in Java, divided into two levels, each of which tackle issues about the Krebs cycle, the respiratory chain and oxidative phosphorylation. Initially, the names of the components of the metabolic process appear randomly on computer screen and the user must follow clues to place them in the right sequence, until all the biochemical reactions are complete. In next phase, there is a quiz about details and clinical correlates related to the theme of the game. Finally, students have to answer a form in order to verify acceptance and relevance of the game. DISCUSSION AND RESULTS: The game was applied to 40 medical students from UFF. The game's subject matter and its difficulty were analyzed and more than $40 \%$ of students classified both respiratory chain and Krebs cycle as difficult. These findings highlight the need to establish new methods to enhance the teaching and learning processes and decrease the students' difficulties, which is the game's purpose. The game was very highly rated by students once they evaluated the game as an excellent educational aid and $92 \%$ of students agreed that it complements the content discussed in classroom. Finally, 97,5\% of students said they would play again. CONCLUSION: Therefore, educational games could be an excellent tool to optimize learning.

Keywords: Game; Metabolism; Teaching 